\title{
Enjeux autour de la pratique enseignante en contexte d'enseignement distancié
}

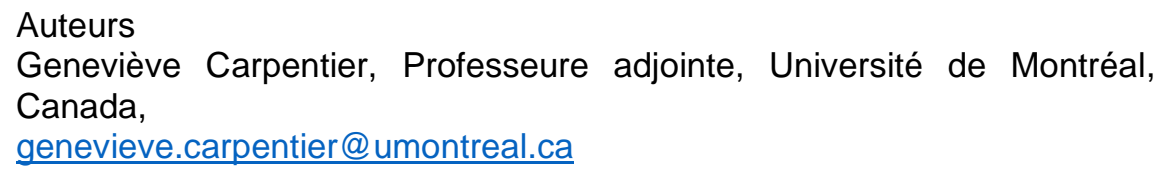

Claudine Sauvageau, Doctorante et chargée de cours, Université de Montréal, Canada,

claudine.sauvageau@umontreal.ca

Normand Roy, Professeur agrégé, Université de Montréal, Canada, normand.roy@umontreal.ca 


\section{$\&$}

\section{REVUE HYBRIDE DE L'ÉDUCATION}

\section{Résumé}

Au primaire, le retour en classe à l'automne 2020, marqué par une distanciation physique entre les élèves et les enseignants.es, pose certains enjeux. Cet enseignement distancié influence notamment la pratique enseignante par l'ajustement des gestes professionnels au regard des diverses préoccupations éducatives, soit le pilotage, l'atmosphère, le tissage, l'étayage et les savoirs visés. Cet article présente les résultats issus d'une recherche descriptive, dont la collecte de données a permis d'interroger dix enseignantes du primaire à propos de l'ajustement de leurs pratiques en contexte d'enseignement distancié. Les résultats révèlent des adaptations prédominantes des pratiques relativement à l'atmosphère et aux savoirs visés.

Mots-clés: pratique enseignante; gestes professionnels; élèves du primaire ; enseignement distancié ; COVID-19 


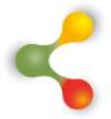

\section{REVUE HYBRIDE DE L'ÉDUCATION}

\section{Introduction et problématique}

La situation inédite du printemps 2020 , en raison de la pandémie de COVID-19, a provoqué plusieurs perturbations dans le monde de l'éducation. Ces dernières ont notamment soulevé de profondes interrogations quant à l'adaptation des modes d'apprentissage et des pratiques d'enseignement (Villiot-Leclercq, 2020). En effet, I'UNESCO recensait au début du mois d'avril 2020 que $91 \%$ de la population scolaire mondiale était affectée par les fermetures d'écoles, menant plus d'un milliard d'élèves à devoir apprendre autrement, et tout.e.s leurs enseignant.e.s, par le fait même, à apprendre à enseigner autrement.

En sol québécois, lorsque la grande majorité des élèves du primaire ont pu réintégrer leur école lors de la rentrée scolaire 2020, l'enseignement en présentiel, balisé par une distance physique à maintenir entre les élèves et les enseignants.es dans les classes, a posé plusieurs nouveaux défis. Ces pratiques ajustées, appelées ici enseignement distancié, s'inscrivent dès lors dans la réalité des classes. L'enseignement distancié au primaire se définit selon divers aspects. Au Québec, il se caractérise notamment par un regroupement des élèves en groupesclasses stables, dont la taille demeure identique à celle des groupes avant la pandémie. Dans le cas de ces groupes, souvent appelés «bulle-classe », la distanciation physique n'est pas requise entre les élèves bien qu'elle le soit entre l'enseignant.e et ses élèves. Plus précisément, les enseignants.es doivent porter du matériel de protection (masque, lunettes, visière) et éviter les contacts rapprochés avec les élèves (de leur groupe ou non) sans ce matériel (gouvernement du Québec, 2020a).

Partant de ces faits, enseigner à des élèves du primaire en tenant compte de ces mesures, en nettoyant entre chaque utilisation le matériel pédagogique et en réduisant le partage d'objets (feuilles, cahiers, crayons, etc.) représente un défi colossal. Ces mesures sanitaires et de distanciation peuvent être génératrices de stress pour les enseignant.e.s et, de surcroit, exiger d'eux.elles de pallier les effets de ces mesures sur leurs élèves. À cet égard, Rezrazi (2020) distingue deux agents anxiogènes qui peuvent affecter psychologiquement les apprenants.es: 1) la propagation de la maladie, 2) les mesures mises en place pour lutter contre la pandémie comme le confinement et l'enseignement distancié. Les enfants en âge de fréquenter le primaire sont donc directement touchés par le contexte de la pandémie. À cet effet, une revue de presse francophone sur la santé mentale liée à la COVID-19 rapporte une augmentation des prescriptions de médicaments contre l'anxiété chez les enfants ainsi qu'une diminution de la motivation et de l'engagement scolaires (Talbot et Lessard, 2020).

À la lumière de ces constats, la réouverture des écoles semble néanmoins représenter un facteur de protection pour la santé mentale des élèves (Talbot et Lessard, 2020), même si d'autres enjeux doivent être considérés en contexte d'enseignement distancié. En effet, l'apprentissage 


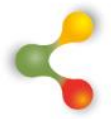

\section{REVUE HYBRIDE DE L'ÉDUCATION}

chez les jeunes élèves de six à douze ans comporte des défis particuliers liés à leur besoin accru de soutien, d'encadrement ainsi qu'aux particularités des pratiques pédagogiques mises en œuvre auprès d'eux, qui nécessitent du matériel de manipulation (Simonin, 2019), des jeux (Wood, 2009), des représentations matérielles des apprentissages faits (Calkins, 2020) ainsi que des interactions sociales entre pairs et avec l'enseignant.e (Laurendeau, 2020; Morlaix, 2015). L'enseignement au primaire est entre autres caractérisé par une grande proximité entre l'enseignant.e et les élèves (Cinéus, 2020), que ce soit pour des motifs pédagogiques (rétroaction individuelle, récupération, rassemblement pour la lecture), pour des raisons liées au développement des élèves (aide à l'habillement et à l'hygiène corporelle) ou dans le cadre d'interactions sociales et émotionnelles (consoler, réconforter, féliciter). II ressort de ces observations que l'enseignement distancié parait préférable à l'enseignement à distance au primaire, notamment puisqu'il entretient le besoin de socialisation essentiel chez les jeunes (Morlaix, 2015). Ainsi, les enseignant.e.s doivent trouver des solutions innovantes pour mettre en œuvre des pratiques pédagogiques favorisant l'engagement, la motivation et le développement de compétences des élèves et contribuant à réduire l'anxiété chez ces derniers (Goyette et al, 2020).

\section{Cadre théorique : La pratique enseignante}

Depuis les dernières décennies, différentes conceptions ont été proposées dans le but de définir la pratique enseignante. Au fil de son évolution, ce concept a notamment été analysé d'un point de vue " écologique " par Bronfenbrenner (1986), qui a introduit l'apport de la situation pédagogique dans sa conception de la pratique enseignante. Des modèles interactionnistes ont par la suite pointé divers types de variables concernant à la fois l'enseignant.e, l'apprenant.e et la situation. C'est le cas d'Altet (2002) qui décrit la pratique enseignante comme « des actions, des interactions et des transactions en situation » (p. 85). Dans le même ordre d'idées, Beillerot (1998) évoque la dimension pédagogique et la dimension didactique de la pratique enseignante. Sous cet angle, la pratique enseignante est considérée comme la manière, propre à un individu, d'effectuer toute activité professionnelle relative à l'enseignement. En ce sens, elle se rapporte tant aux actes observables, qu'aux procédés employés, qu'aux prises de décisions sous-jacentes lors de la mise en œuvre d'une activité dans une situation donnée (Altet, 2002 ; Beillerot, 1998). La pratique enseignante, qui nécessite des ajustements, entre autres selon le contexte, les apprenants.es et le contenu, "s'organise en une architecture dense de gestes professionnels (Bucheton, 2009, p. 54). Le choix de ces gestes professionnels est étroitement lié à la volonté des enseignants.es d'adapter leurs actions aux évolutions de la situation éducative, voire au contexte vécu (Maubant, 2007). C'est d'ailleurs ce que le référentiel québécois des compétences professionnelles de la profession enseignante met en relief en misant notamment sur les capacités de 


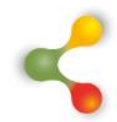

\section{REVUE HYBRIDE DE L'ÉDUCATION}

raisonnement, de jugement et de réflexion des enseignants.es selon le contexte d'intervention (gouvernement du Québec, 2020b).

Beckers (2007) précise qu'il s'agit d'ailleurs d'une caractéristique propre aux métiers basés sur l'interaction humaine : ajuster les pratiques et les gestes professionnels selon les acteurs.trices en jeu et le contexte. C'est aussi ce que Bucheton (2009) désigne comme l'empan d'ajustement. En effet, il est possible d'ajouter à ces adaptations celles, non négligeables, imposées par les évolutions technologiques et sociétales. Dans cette perspective, bien que la pratique enseignante soit effectivement en constante adaptation, le contexte de la pandémie mondiale semble avoir fortement secoué les pratiques (Villiot-Leclercq, 2020). En effet, la gestion d'un changement d'envergure se planifie généralement longtemps à l'avance. Or, dans le contexte inédit de la pandémie, l'ensemble du système d'éducation a dû s'adapter très rapidement et de façon considérable (Bissonnette, 2020).

Dans l'intention de mieux comprendre les gestes professionnels des enseignants.es, qui doivent s'ajuster à une diversité de situations, Bucheton et Soulé (2009) proposent le modèle de multi-agenda. Celui-ci rend compte de la manière dont les gestes professionnels peuvent générer différentes dynamiques dans la classe. Le multi-agenda s'articule autour de cinq préoccupations, jamais isolées les unes des autres, qui constituent la matrice de la pratique enseignante. 1) Le pilotage de la leçon renvoie à ce qui est mis en œuvre pour assurer la cohérence et la cohésion de toute séance d'enseignement. 2) L'atmosphère se rapporte à tout ce qui permet d'organiser la rencontre intellectuelle, relationnelle, affective et sociale entre les différent.e.s acteurs.trices confronté.e.s à une situation commune. 3) Le tissage fait référence à l'activité de l'enseignant.e ou des élèves pour établir des liens visant la contextualisation, la décontextualisation et la recontextualisation. 4) L'étayage représente l'aide apportée aux élèves par l'enseignant.e pour favoriser notamment l'action, la pensée et la compréhension. 5) Les savoirs visés constituent la cible d'une situation d'enseignement-apprentissage donnée.

Précisons néanmoins que le basculement vers l'enseignement distancié entraine des ajustements non seulement face aux situations pédagogiques et didactiques, mais également sur le plan des relations interpersonnelles et de l'environnement physique en respect des mesures sanitaires et de distanciation en vigueur. Si ces ajustements demeurent peu documentés, d'où la pertinence de mener ces travaux, il est possible de s'appuyer sur ce que la littérature scientifique avance au sujet du rôle de premier plan que joue la pratique enseignante sur la réussite et le bienêtre des élèves. À cet égard, Hattie (2017) met en exergue l'effet enseignant sur les apprentissages des élèves. De ce point de vue, l'enseignant.e exercerait notamment une influence positive sur la réussite des élèves en fixant des objectifs ambitieux et en les invitant à relever ces défis. De même, Archambault (2009) prône les pratiques enseignantes qui favorisent 


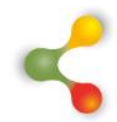

\section{REVUE HYBRIDE DE L'ÉDUCATION}

l'autonomie des élèves, mettent l'accent sur la valorisation des apprentissages et considèrent les besoins et les intérêts des élèves. II semble que les résultats découlant de telles pratiques conduiraient les élèves à s'investir, à oser commettre des erreurs, à développer leurs compétences et à saisir la pertinence des apprentissages effectués (Archambault, 2009 ; Hattie, 2017 ; Schwartz, 2016).

De plus, la qualité de la relation enseignant.e-élève et le soutien pédagogique auraient une influence sur le bienêtre des élèves en classe. Sur ce point, Baudoin et Galand (2018) soulèvent l'intérêt de pratiques enseignantes basées sur la mise en place de routines, sur le suivi des progrès des élèves et des interactions sociales entre élèves ainsi que sur l'établissement de règles claires. De surcroit, la création d'un environnement bienveillant en classe dans lequel les élèves évoluent aurait un effet positif sur eux, tant sur le plan du bienêtre physique, cognitif que psychosocial (Goyette et al., 2020), et limiterait les comportements négatifs émergents (Gaudreau et al., 2016). En somme, si l'influence de la pratique enseignante sur la réussite et le bienêtre des élèves est reconnue, tout porte à croire que son apport se poursuit en contexte d'enseignement distancié, teinté de certains ajustements. Dans cette perspective, cet article vise à décrire et à comprendre les enjeux de l'enseignement distancié sur les gestes professionnels d'enseignants.es du primaire.

\section{Méthodologie}

Une recherche descriptive a été privilégiée puisque celle-ci vise à décrire et à analyser en profondeur des comportements, des évènements et des phénomènes peu connus, dans un environnement en particulier (Thouin, 2014). Dans notre cas précis, la description porte sur les enjeux de l'enseignement distancié en contexte de pandémie. De même, puisque nous cherchons à comprendre ces enjeux du point de vue des enseignants.es qui vivent l'enseignement distancié, notre recherche prend également un angle phénoménologique (Ribau et al., 2005).

\section{Les participant.e.s et le déroulement}

La collecte de données a eu lieu à l'automne 2020 auprès d'enseignantes $(n=10)$ des trois cycles du primaire, venant du Centre de services scolaire des Affluents (5) et du Centre de services scolaires des Samares (5). II s'agit d'enseignantes d'expérience variée (de 3 à 28 années d'expérience) qui œuvrent au $1^{\mathrm{er}}(\mathrm{n}=4)$, au $2^{\mathrm{e}}(\mathrm{n}=3)$ ainsi qu'au $3^{\mathrm{e}}$ cycle du primaire $(n=3)$. Des entretiens semi-dirigés (Gauthier, 2016), essentiellement fondés sur le modèle du multi-agenda de Bucheton et Soulé (2009), ont été effectués. Dans cette perspective, les enseignantes étaient invitées à s'exprimer à propos de l'ajustement des cinq gestes professionnels à la base de ce modèle (le pilotage, l'atmosphère, le tissage, l'étayage et les savoirs) en contexte d'enseignement distancié. Chacun des gestes professionnels a été défini pour faciliter leur compréhension et 


\section{6}

\section{REVUE HYBRIDE DE L'ÉDUCATION}

guider leurs réflexions (Royer et al., 2009). L'analyse thématique de Paillé et Mucchielli (2016) a été utilisée pour analyser les données issues des entretiens puisqu'elle permet une " transposition d'un corpus donné en un certain nombre de thèmes représentatifs du corpus analysé, et ce, en rapport avec l'orientation de la recherche» (p. 236) qui, dans notre cas précis, réfère aux enjeux de l'enseignement distancié. Un premier codage a été effectué selon les cinq gestes professionnels. En observant le résultat de ce premier codage, il est apparu que certains propos semblaient ressortir de façon plus importante selon le cycle d'enseignement des enseignantes. Un second codage a donc été réalisé au regard des cycles d'enseignement afin d'affiner notre compréhension.

\section{Résultats et discussion}

Rappelons que l'objectif de cet article porte sur la description des gestes professionnels d'enseignantes en contexte d'enseignement distancié. Le modèle du multi-agenda de Bucheton et Soulé (2009) a guidé les entretiens et leur analyse ; nous présentons donc les observations des enseignantes selon les composantes de ce modèle.

\section{Les savoirs visés}

Les enseignantes du $1^{\text {er }}$ cycle sont celles qui ont le plus modifié et adapté leur planification annuelle. C'est particulièrement l'apprentissage de la langue qui a monopolisé la discussion. L'un des effets majeurs de la pandémie sur leurs pratiques concerne l'absence ou la diminution de l'enseignement de la conscience phonologique auprès des élèves de la maternelle et du travail d'automatisation des associations graphèmesphonèmes auprès des élèves de $1^{\text {re }}$ année au printemps 2020. Les enseignantes du $1^{\mathrm{er}}$ cycle ont donc senti le besoin de travailler davantage les habiletés de prélecture et de préécriture lors du retour en classe à l'automne 2020. Cet ajustement paraissait nécessaire afin de respecter le rythme et les connaissances antérieures des élèves :

Habituellement, en début d'année, la plupart de mes élèves de $1^{\text {re }}$ année connaissent le son de plusieurs lettres. Cette année, j'ai vite compris que ce n'était pas le cas et que je devais prendre vraiment plus mon temps pour respecter leur développement (enseignante E2).

Sur le plan des savoirs visés, un premier constat général porte donc sur l'importance prédominante accordée par les enseignantes interrogées au domaine disciplinaire de la langue. En effet, les principales considérations qui ressortent portent d'abord sur la lecture, et dans une moindre mesure, sur l'écriture. En sachant que la compétence et l'appétence (liée au plaisir) des élèves en lecture sont des éléments centraux et prédicteurs de leur réussite scolaire, sociale et culturelle (Lépine, 2017), le choix des enseignantes de centrer leur effort sur cette 


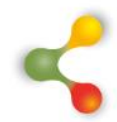

\section{REVUE HYBRIDE DE L'ÉDUCATION}

matière peut avoir du sens. Au Québec, en temps normal, c'est environ le quart des élèves du $3^{\mathrm{e}}$ cycle du primaire qui n'atteignent pas le niveau de compétence en lecture requis pour accéder au secondaire (ministère de l'Éducation, du Loisir et du Sport, 2011). II semble donc y avoir une sorte de priorisation des savoirs par les enseignantes en fonction du retard accumulé, mais aussi en fonction de la place centrale que joue la lecture dans la réussite globale des élèves.

Par ailleurs, si la planification annuelle en lien avec les savoirs à développer des six enseignantes des $2^{\mathrm{e}}$ et $3^{\mathrm{e}}$ cycles n'a pas été considérablement modifiée, ces dernières ont effectué des retours plus approfondis des connaissances antérieures de leurs élèves avant d'entamer de nouveaux apprentissages. À cet effet, les enseignantes remarquent que les élèves ont plus de difficulté à accéder aux apprentissages réalisés l'année précédente :

Les notions sont loin. II faut travailler longtemps, sortir notre matériel de manipulation, retourner voir nos collègues des niveaux antérieurs et s'armer de patience. Pour travailler les multiplications cette année, en $5^{\mathrm{e}}$ année, j'ai ressorti du matériel de base dix (enseignante E8).

Pour cinq enseignantes, ce constat se révèle assez positif par le fait qu'elles questionnent davantage les élèves pour évaluer leur niveau de connaissance et ainsi offrir un enseignement davantage centré sur leurs besoins. Deux d'entre elles mentionnent toutefois que cette situation accentue considérablement les écarts entre les élèves.

Pendant que je prends une période complète pour revenir sur les notions liées au verbe, au prédicat, à la terminaison et aux personnes, j'ai les trois quarts de mes élèves qui font plein de liens et qui récupèrent des notions qu'ils avaient oubliées ou jamais vraiment comprises et j'ai le quart de mes élèves qui savent déjà tout ça et trouvent ça plate (enseignante E10).

De ces constats émergent divers ajustements de la pratique enseignante pour optimiser le temps d'enseignement. Par exemple, les deux enseignantes de $3^{\mathrm{e}}$ année précisent que leur école a choisi de ne pas miser sur l'apprentissage de l'écriture cursive lors du retour en classe à l'automne 2020 : «L'écriture cursive, c'est le seul gros apprentissage que nos élèves n'auront pas fait à cause de la pandémie. Nous avons choisi de simplement le mettre de côté et de nous concentrer sur le reste" (enseignante E6). D'autres décisions pédagogiques ont été prises dans les équipes-écoles pour faciliter une possible transition à l'enseignement à distance. C'est le cas des six enseignantes des $2^{\mathrm{e}}$ et $3^{\mathrm{e}}$ cycles qui ont effectué l'achat de cahiers d'exercices. Or, selon les quatre enseignantes 


\section{8}

\section{REVUE HYBRIDE DE L'ÉDUCATION}

qui n'avaient pas l'habitude d'utiliser ce type de matériel didactique, l'expérience s'avère plutôt négative : "C'est très contraignant, il faut sans cesse adapter ce qui est proposé ou voir le contenu dans un ordre différent » (enseignante E7). L'ordre d'enseignement du contenu semble d'ailleurs un aspect central de l'ajustement des pratiques pour plusieurs enseignantes dans le contexte actuel. Par exemple, l'enseignante E10 entame habituellement l'année scolaire en travaillant les textes informatifs. Cependant, en ressentant l'état anxieux de plusieurs élèves ainsi que les émotions fortes et intenses qu'ils vivaient lors de la rentrée scolaire 2020, elle a plutôt choisi de commencer l'année par un module sur la poésie :

Je trouve que ce choix m'a permis d'entrer en action en douceur. J'ai pu faire lire des œuvres aux élèves qui parlaient du stress, du doute, etc. Ils ont pu s'exprimer librement en poésie et cela m'a permis de les connaitre et de tisser rapidement des liens avec eux (enseignante E10).

Les entretiens auprès des enseignantes démontrent donc l'accent mis sur l'ajustement de la planification de l'enseignement. Ces adaptations rappellent l'empan d'ajustement, comme décrit par Bucheton (2009). Elles se fondent sur le souci de suivre le rythme d'apprentissage des élèves, en prenant notamment en considération leurs connaissances antérieures (Barth, 2004) et leur zone proximale de développement (Vygotski, 1997). En d'autres termes, elles enseignent ce que les élèves sont prêts à apprendre (Schwartz, 2016). Or, dans un contexte instable de pandémie, caractérisé par le basculement d'un enseignement à distance vers un enseignement distancié, les entretiens des enseignantes révèlent que la disponibilité et l'accessibilité aux apprentissages reposent également sur la prise en compte des émotions des élèves. De ce fait, une entrée par les œuvres littéraires, voire par la poésie, comme l'a fait l'enseignante E10, représenterait une avenue susceptible de favoriser les apprentissages, par une ouverture naturelle aux émotions et à leur partage (Florey et Cordonier, 2017 ; gouvernement de l'Île-du-Prince-Édouard, 2006).

Selon les enseignantes interrogées, l'ajustement de la planification de l'enseignement s'effectue également au regard des écarts entre élèves. Si les pratiques de différenciation pédagogique pour réduire ces écarts sont relativement bien documentées en recherche (Auclair Tourigny, 2017; Villeneuve-Lapointe et al., 2018), il semble que l'accentuation des retards scolaires accumulés par nombre d'élèves constitue un phénomène propre au contexte scolaire de l'automne 2020 et exige une souplesse pédagogique accrue. En effet, Rasinski et Padak (2004) ont déjà mis en évidence les oublis et les régressions des élèves du primaire d'une année scolaire à une autre après les deux mois de vacances d'été. En fluidité et en compréhension de lecture, par exemple, ces chercheurs ont mis en lumière que les élèves retrouvent leur niveau de lecture acquis à la fin de leur précédente année scolaire au milieu de l'année scolaire suivante. Dans 


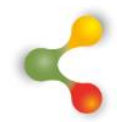

\section{REVUE HYBRIDE DE L'ÉDUCATION}

la situation actuelle, avec une scolarisation très variable entre les élèves pendant plus de cinq mois, les enseignantes observent un retard accru pour plusieurs élèves qui nécessite des ajustements des notions présentées, des retours sur les connaissances plus approfondis et un respect de leur rythme d'apprentissage. Cette souplesse et l'adaptation dont ont témoigné les enseignantes laissent croire que le soutien pédagogique (Baudoin et Galand, 2018) et les pratiques centrées sur les besoins des élèves (Archambault, 2009) sont centraux dans leurs gestes professionnels.

\section{L'atmosphère}

Cette composante semble être la plus influencée par l'enseignement distancié. En effet, sept enseignantes ont mentionné que leurs élèves paraissaient plus anxieux, moins confiants et moins motivés. À cet égard, il importe de préciser que ces observations proviennent principalement des enseignantes du $2^{\mathrm{e}}$ et du $3^{\mathrm{e}}$ cycle. Dans ces conditions, elles ont investi plus de temps dans les discussions en grand groupe portant sur le vécu et les questionnements des élèves. Cette sensibilité, issue de l'ajustement des pratiques, serait d'ailleurs à conserver selon plusieurs enseignantes : " Je n'ai jamais autant pris le temps de connaitre mes élèves, de leur demander si le fonctionnement de la classe leur convient. Je les implique beaucoup plus qu'avant et c'est quelque chose que j'aimerais garder après la pandémie » (enseignante E7). Les enseignantes du $1^{\text {er }}$ cycle ont également relevé des enjeux motivationnels, mais elles soulignent que les enjeux liés à la concentration, à l'attention et à une certaine fatigue cognitive influencent encore plus l'atmosphère de leur classe.

Le sentiment d'anxiété des élèves, influençant l'atmosphère de la classe, semble souvent directement lié à une peur de propagation de la maladie. Ainsi, les élèves pouvaient être très nerveux en début d'année dès qu'un autre élève toussait : " II a fallu dédramatiser un peu la situation et se rappeler qu'il est possible qu'un ami éternue sans avoir la COVID$19 »$ (enseignante E6). De même, plusieurs élèves s'inquiètent de l'absence de leurs camarades en classe, qu'elle soit liée ou non aux symptômes de la COVID-19:

Par exemple, si un élève est absent pendant deux ou trois jours, c'est certain que les élèves qui sont dans le même ilot de travail que lui vont venir me demander s'il a la COVID, s'ils doivent aller se faire tester. J'ai même des parents qui m'écrivent pour ça toutes les semaines (enseignante E3).

L'atmosphère de la classe semble donc au cœur des préoccupations des enseignantes en cette période d'instabilité. Le vécu de classe qu'elles relatent souligne effectivement l'influence marquée de 


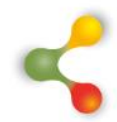

\section{REVUE HYBRIDE DE L'ÉDUCATION}

l'enseignement distancié et du contexte de pandémie sur l'anxiété des élèves. À cet égard, Papazian-Zohrabian et Mamprin (2020) rappellent "que la COVID-19 a rendu soudain la "mort intime", a augmenté le potentiel de pertes (deuils) et de ruptures (traumatismes), en plus de mettre à rude épreuve la santé mentale de tous » (p. 6). Les réactions des élèves lors de l'apparition de symptômes liés à la COVID-19 chez leurs camarades de classe ou lors de l'absence d'un de ces derniers sont donc normales. La santé mentale, la capacité d'adaptation ainsi que les apprentissages scolaires des élèves sont influencés par l'augmentation potentielle de traumatisme ou de deuil, par le confinement prolongé et par la distanciation sociale et physique (Rezrazi, 2020). Les conséquences probables de la pandémie sur les élèves documentés par la recherche scientifique comme les problèmes d'autorégulation, les difficultés de concentration, la désorganisation, la fatigue, la démotivation ainsi que le manque de disponibilité cognitive pour les tâches scolaires (Papazian-Zohrabian et Mamprin, 2020) sont ressortis dans les dix entretiens menés dans le cadre de ce projet.

Dans un autre ordre d'idées, l'instauration des bulles-classes agit sur différents plans de l'atmosphère de la classe. Alors que quatre enseignantes estiment que la dynamique de leur groupe est particulièrement intéressante cette année - « II arrive que tous mes élèves jouent ensemble à la récréation. Je n'ai jamais vu ça » (enseignante E9.) -, les six autres enseignantes évoquent plutôt la lourdeur de gestion de la bulle-classe. Parmi celles-ci, trois enseignantes du $1^{\text {er }}$ cycle soulignent que "l'effet bulle » favorise les conflits et empêche les élèves d'avoir une "pause » pendant les récréations. Les conflits semblent aussi accentués par le fait que les enseignantes doivent demeurer avec leurs élèves lors des déplacements :

Habituellement, lorsque mes élèves sont habillés pour la récréation, je les envoie tranquillement dehors. Maintenant, je ne peux plus faire ça, je dois aller moimême les reconduire lorsqu'ils sont tous prêts. II y a donc des élèves qui attendent très longtemps après les autres et qui se plaignent d'avoir chaud et de manquer du précieux temps de récréation (enseignante $\mathrm{E} 1$ ).

En accord avec ces constats, d'autres enseignantes ajoutent que l'orchestration des déplacements dans l'école, pour respecter les normes de sécurité, a affecté la durée de certaines récréations. À cet effet, l'enseignante E2 mentionne :

À mon école, la récréation dure 14 minutes. Je suis en $1^{\text {re }}$ année. Si je ne sors pas d'avance pour que tous mes élèves s'habillent, ils n'auront pas de récréations. Ça crée de la frustration chez les enfants lorsque j'oublie de sortir d'avance et ça me met un stress supplémentaire. 


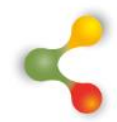

\section{REVUE HYBRIDE DE L'ÉDUCATION}

En ce qui a trait aux contacts physiques entre les élèves et leur enseignante, les dix enseignantes affirment qu'il est possible de les limiter, mais qu'il est impossible de les éliminer complètement. Pour cette raison, huit des enseignantes interrogées déclarent porter le matériel de protection (masque, lunettes ou visière) en tout temps dans la classe, même quand elles sont à plus de deux mètres des élèves :

Au début de l'année, il m'arrivait d'enlever mon matériel de protection quand j'étais, par exemple, à mon bureau ou devant la classe pendant un moment. Par contre, si un élève se levait rapidement ou s'il arrivait à mon bureau sans faire de bruit, je faisais le saut et je paniquais un peu en lui disant de ne surtout pas bouger pendant que j'enfilais en vitesse mon masque et mes lunettes. Parfois, ça faisait faire le saut aux élèves ou ça brisait le moment (enseignante E5).

Une autre enseignante tente de maintenir une distance en tout temps avec ses élèves, mais elle sent que ça affecte sa relation avec eux : «Des fois, ce n'est pas grand-chose, une main sur l'épaule, une brève accolade, un high five ça solidifie le lien. Cette année, ça me manque et je sens que ça manque aussi à mes élèves " (enseignante E6). Pour les enseignantes du $1^{\text {er }}$ cycle, le contact physique semble plus important en raison de l'âge de leurs élèves :

Mes cocos sont petits, plusieurs ont besoin de contacts physiques pour les réconforter, mais encore plus en ont besoin pour les aider. La COVID et les mesures sanitaires n'enlèvent pas les manteaux à zipper, les tuques à attacher et les mitaines à enfiler (enseignante E2).

À cet égard, si le regroupement des élèves en bulles-classes renforce les relations entre élèves dans certaines classes, il exacerbe les comportements négatifs et les conflits entre élèves dans plusieurs autres. Le peu de contacts sociaux que les élèves ont eu pendant de longs mois peut potentiellement expliquer ces résultats. Laurendeau (2020) estime qu'il ne faut pas ignorer les effets qu'a eus le confinement sur le sentiment collectif d'isolement et d'anxiété. En effet, la qualité des relations interpersonnelles peut être affectée par l'anxiété, nuisant au développement des habiletés sociales (Lambert-Samson, 2016). Dans cette optique, les enseignantes interrogées misent sur la création d'un environnement ouvert et bienveillant, qui laisse place aux questionnements des élèves, comme au partage de leur vécu et de leurs émotions, et qui tient compte de leur avis concernant le fonctionnement de la classe. Selon Goyette et al. (2020), un tel environnement bienveillant aurait un effet positif sur les élèves, tant sur le plan du bienêtre physique, cognitif que 


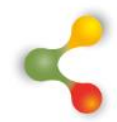

\section{REVUE HYBRIDE DE L'ÉDUCATION}

psychosocial. Qui plus est, un climat de classe sécurisant et une relation enseignant.e-élève positive permettraient d'agir sur l'anxiété des élèves comme sur les comportements négatifs émergents, et par conséquent, sur l'atmosphère de la classe (Gaudreau et al., 2016). II semble d'ailleurs que la relation enseignant.e-élève soit centrale dans le contexte scolaire actuel, possiblement en raison du peu de compromis que les enseignantes font à cet égard, même en enseignement distancié. Entre autres, les contacts physiques entre les élèves et elles demeurent généralement inévitables, tant pour des motifs pédagogique, développemental, social ou émotionnel (Cinéus, 2020).

\section{Le pilotage des activités}

Cette dimension semble moins affectée par le contexte d'enseignement distancié. Malgré cela, trois enseignantes, qui ont choisi de ne plus aménager de coin de rassemblement dans leur classe, considèrent que leur pilotage est moins "actif» qu'il l'était les années précédentes. De même, l'utilisation de cahiers d'exercices par des enseignantes qui travaillent généralement sans ce matériel est relevée comme un mauvais compromis, nuisant à leur pilotage : "Je trouve que mes pratiques pédagogiques sont moins variées cette année. Je me sens prisonnière de mes cahiers d'activités et je fais moins de projets, d'enseignement inductif et d'apprentissage par problèmes " (enseignante E4). Huit enseignantes ont mentionné que le manque de temps les amène à privilégier un enseignement plus «direct » (enseignement magistral, enseignement explicite, exposé). Trois d'entre elles se questionnent sur la pertinence de ce choix :

\begin{abstract}
Je fais beaucoup plus d'enseignement magistral qu'avant pour gagner du temps, mais j'ai l'impression que le temps que je gagne, je dois le réinvestir en revenant constamment sur les mêmes notions. Au final, je crois que je devrais revenir à des types d'enseignement qui prennent plus de temps, mais qui semblent plus efficaces (enseignant E6).
\end{abstract}

Les enseignantes qui travaillaient déjà avec un cahier semblent néanmoins constater des différences moindres dans le pilotage des tâches.

L'achat de cahiers d'exercices, dans le but de faciliter une potentielle transition vers l'enseignement à distance, semble donc affecter le pilotage des activités pédagogiques. Les enseignantes qui avaient délaissé l'utilisation de cahiers en classe se sentent contraintes à les réintégrer à leur pratique. Dès lors, celles-ci souhaitent se doter d'une autonomie vis-à-vis du contenu des cahiers et les utiliser de manière sélective, en complément à d'autres types d'activités, ce que Boutonnet (2018) décrit comme un usage extensif du matériel didactique. Malgré cela, plusieurs enseignantes se sentent coincées par le temps accordé à 


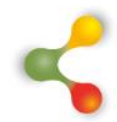

\section{REVUE HYBRIDE DE L'ÉDUCATION}

l'utilisation de ce matériel, mais également par l'adaptation de leurs pratiques « habituelles », notamment pour rattraper les retards scolaires. En conséquence, une centration sur les «matières de base " (français et mathématique) et une perte de repères sont observables : les pratiques innovantes s'effritent et les anciens repères prennent le dessus, se caractérisant par des méthodes d'enseignement plus transmissives et magistrocentrées. Une telle situation peut s'expliquer par la déconstruction des habitus des enseignantes, empreintes de questionnements, conduisant à considérer cette période comme une parenthèse (VilliotLeclercq, 2020), durant laquelle un retour temporaire à des pratiques plus «traditionnelles» ou du moins, qui donnent plus de contrôle à l'enseignante, se montre rassurant.

\section{L'étayage}

Selon les enseignantes, ce n'est pas l'enseignement distancié qui influence l'étayage qu'elles fournissent, mais plutôt le retard scolaire que certains élèves ont accumulé et qui continue de s'accentuer, augmentant par le fait même les écarts entre les élèves, depuis le printemps 2020.

Je sais qu'à la maison, on fait plein d'autres apprentissages importants comme se faire à manger, mais ça reste que pendant plusieurs mois, j'ai des élèves qui ont joué à des jeux vidéo plusieurs heures par jour, qui ont remplacé l'école et leur vie sociale par les écrans, qui ont dû arrêter le sport... Ça laisse des traces sur eux, sur leur savoir-être... Ça accentue les écarts entre les élèves qu'on le veuille ou non (enseignante E5).

Pour sept enseignantes, l'étayage prend donc une place prépondérante cette année, mais elles ont l'impression de " couper » sur d'autres matières :

Habituellement, à ce stade de l'année, j'ai fait trois grands projets en arts et les élèves ont fait plusieurs expériences scientifiques. Cette année, je fais des deuils de certains projets en sachant que ce sont souvent de ceux-là dont mes élèves vont se souvenir longtemps (enseignante E9).

Ainsi, pour ces enseignantes, les retards à rattraper pour certains élèves sont tels qu'elles doivent prendre beaucoup plus de temps pour modéliser, pour manipuler et pour faire des pratiques variées (guidées, coopératives, autonomes). Le temps additionnel qu'elles investissent pour l'étayage de notions en français et en mathématique enlève du temps pour les autres matières et pour des projets de plus grande envergure. L'enseignante E5 mentionne également sur le même sujet : 


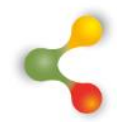

\section{REVUE HYBRIDE DE L'ÉDUCATION}

Je dois prendre plus de temps cette année pour enseigner plus en profondeur certaines notions et je coupe sur les projets en univers social qui font habituellement le plus «tripper» mes élèves. J'ai l'impression de ne pas avoir le choix, mais c'est quand même difficile (enseignante E5).

Ces deux enseignantes affirment néanmoins qu'elles en parlent ouvertement avec leurs élèves afin qu'ils comprennent la situation.

Toujours en ce qui a trait à l'étayage, le tutorat des élèves plus vieux, instauré dans les écoles avant la pandémie, manque particulièrement aux enseignantes du $1^{\mathrm{er}}$ cycle. Les quatre enseignantes ont fait remarquer qu'elles ne peuvent plus faire de jumelage entre des lecteurs débutants et des lecteurs experts du $2^{\mathrm{e}}$ ou du $3^{\mathrm{e}}$ cycle : " Je m'ennuie vraiment de mon escouade d'experts. En plus de valoriser les plus vieux, cette pratique permettait vraiment d'augmenter le nombre de pratiques de lecture à voix haute de mes élèves » (enseignante E1). Ces pratiques de tutorat sont également regrettées par une des enseignantes de $3^{\mathrm{e}}$ cycle qui trouve que ces élèves étaient valorisés par l'aide qu'ils apportaient aux plus petits et que certains développaient même de belles complicités avec leur tutoré.e. Dans ce même ordre d'idées, cinq enseignantes ont signalé l'impossibilité d'avoir recours à l'aide des parents et des grands-parents, notamment pour la bibliothèque, pour des activités spéciales en classe et pour du soutien aux élèves, qui permettait de leur offrir un meilleur étayage : "Habituellement, un vendredi sur deux, il y a des parents et des grands-parents qui viennent jouer au Scrabble avec mes élèves. C'est un moment précieux où mes élèves travaillent le lexique orthographique en s'amusant » (enseignante E9).

Ces adaptations et ces choix pédagogiques, comme mentionnés précédemment, visent notamment à pallier des retards scolaires qui semblent accentués par le contexte actuel. Dans ce cas, selon le modèle de la diversité des postures d'étayage de Bucheton et Soulé (2009), les enseignantes tiennent davantage une posture d'accompagnement et d'enseignement auprès des élèves à risque. La posture d'accompagnement relève d'une aide ponctuelle, individuelle ou collective, en fonction de l'avancée de la tâche et des obstacles à surmonter, tandis que la posture d'enseignement mène à formuler et à structurer les savoirs et les normes. II ressort des entretiens avec les enseignantes que la posture d'accompagnement favorise la relation entre elles et les élèves et peut également impliquer une relation aidant.e-aidé.e entre d'autres acteurs.trices (Bantigny, 2017). Par exemple, le tutorat entre pairs, fondé sur l'entraide et la coopération, permet la progression mutuelle dans un domaine d'apprentissage, alors que le mentorat favorise le transfert de connaissances et d'expériences (Clark et Andrews, 2009). Si ces relations aidant.e-aidé.e peuvent exister à l'intérieur des bulles-classes, il semble qu'un pan important de ces relations d'aide soit évacué en enseignement 


\section{6}

\section{REVUE HYBRIDE DE L'ÉDUCATION}

distancié : l'apport de collaborateurs.trices de l'extérieur. Ainsi, le tutorat par des pairs plus âgés de l'école ou le mentorat avec des parents ou des grands-parents en classe devient difficile, voire inexistant. Pourtant, maint.e.s chercheurs.euses reconnaissent les avantages cognitifs et socioaffectifs de ces pratiques (Paratte, 2006 ; Potvin et al., 2012).

\section{Le tissage}

Cette dimension n'est pas particulièrement ressortie dans les entretiens menés. Concernant cet aspect, l'enseignante E10 affirme : « Je pense que je fais autant de liens entre les savoirs qu'avant, je prends plus de temps pour expliquer et je reviens plus longuement sur les notions, mais les liens sont toujours les mêmes ". Puisque plusieurs enseignantes ont mis de côté les projets multidisciplinaires ainsi que les projets de grande envergure en arts, en science, en univers social et en éthique et culture religieuse pour prendre plus de temps en mathématique et en français, il est possible de penser que le tissage est un peu plus limité étant donné que cette dimension se déploie plus naturellement dans des projets qui mettent de l'avant l'interdisciplinarité. En effet, ce type de projets occupe un créneau important dans les classes en favorisant la création de liens interdisciplinaires. Se pourrait-il que l'environnement éducatif actuel de la classe engage moins les élèves à tisser des liens entre les disciplines ? Certes, il est crucial de se poser la question, car au-delà de tisser des liens entre les savoirs, les travaux interdisciplinaires motivent les élèves, permettent une variété d'approches et de modes de construction des activités et éclairent de manière informée les phénomènes étudiés (Reuter, 2019). Ce constat ressort également des entretiens et plusieurs enseignantes attestent que les projets évacués pour prioriser les matières de base sont souvent ceux qui sont les plus appréciés par leurs élèves.

\section{Conclusion}

En somme, l'enseignement distancié semble être un enjeu important chez les enseignantes du primaire interrogées; elles ont grandement modifié leur pratique enseignante ainsi que leurs gestes professionnels afin de les adapter et de poursuivre le développement des compétences disciplinaires, sociales et affectives de leurs élèves. Les principaux enjeux qui ressortent sont d'abord liés à l'atmosphère de la classe et aux pratiques mises en place pour diminuer le stress et l'anxiété des élèves, ainsi que les désagréments de la bulle-classe. Les choix liés aux notions à enseigner prennent également une place importante puisque les enseignantes passent plus de temps à étayer certaines notions au détriment de certaines autres.

À ce sujet, plusieurs enseignantes croient que l'enseignement à distance prodigué au printemps 2020 n'a ni permis à la majorité des élèves de faire de nouveaux apprentissages, ni de conserver leurs acquis, accentuant lourdement les écarts entre les élèves. Le type d'enseignement 


\section{$\&$}

\section{REVUE HYBRIDE DE L'ÉDUCATION}

dont font mention les enseignantes est nommé « enseignement à distance d'urgence " ou emergency remote teaching (Laurendeau, 2020) et est caractérisé par un accompagnement minimal des élèves dont l'objectif principal vise le maintien des acquis (Hodges et al., 2020). Ce type d'enseignement, utilisé en temps de pandémie, de catastrophes naturelles, de guerres ou de conflits locaux, devrait prioriser le bienêtre et le plaisir afin, notamment, de diminuer le niveau d'anxiété des élèves (Bozkurt et Sharma, 2020). Les entretiens que nous avons menés semblent confirmer que c'est ce type d'enseignement qui a été mis en place au Québec au début de la pandémie. Ainsi, la majorité des ajustements effectués par les enseignantes réfère aux retards scolaires accumulés ou accentués par le confinement du printemps 2020.

Ces nombreux ajustements ont été effectués dans un contexte marqué par des agents anxiogènes liés à la pandémie (Rezrazi, 2020), qui peuvent certes influencer le bienêtre, la motivation et l'engagement des élèves (Talbot et Lessard, 2020), mais aussi le bienêtre psychologique au travail des enseignant.e.s (Dagenais-Desmarais, 2010). À ce sujet, Papazian-Zohrabian et Mamprin (2020) évoquent que la perception de reconnaissance au travail en contexte de pandémie est complexe : «En effet, certains repères relatifs au travail sont bousculés et il peut être difficile d'observer l'investissement et les bons coups des enseignants et des autres membres du personnel scolaire » (p. 20). En ce sens, nos travaux permettent finalement de mettre en valeur l'adaptation et les ajustements mis en place par des enseignantes du primaire qui ont à cœur le bienêtre et la réussite scolaire et sociale de leurs élèves. 


\section{$\&$}

\section{REVUE HYBRIDE DE L'ÉDUCATION}

\section{Références}

Altet, M. (2002). Une démarche de recherche sur la pratique enseignante : l'analyse plurielle. Revue française de pédagogie, 138, 85-93. https://doi.org/10.3406/rfp.2002.2866

Archambault, I. (2009). Effets de l'environnement scolaire, des attitudes, compétences et pratiques des enseignants sur l'engagement des garçons et des filles en milieux défavorisés. Contributions directes et indirectes (FRQSC) pour l'Université de Montréal. Université de Montréal. http://www.frasc.gouv.qc.ca/fr/la-recherche/la-rechercheen-vedette/histoire/effets-de-l-environnement-scolaire-des-attitudescompetences-et-pratiques-des-enseignants-sur-l-engagement-desgarcons-et-des-filles-en-milieux-defavorises-contributions-directes-etindirectes-h67pxojs1428418896073

Auclair Tourigny, M. (2017). Besoins de soutien des enseignants du primaire québécois : analyse thématique de leurs perceptions en vue d'offrir des pistes d'amélioration pour quatre aspects de leur pratique professionnelle [thèse de doctorat, Université de Sherbrooke]. Savoirs UdeS. https://savoirs.usherbrooke.ca/handle/11143/11067?show=full

Barth, B.-M. (2004). Le savoir en construction. Retz.

Bantigny, R. (2017). Tutorat entre pairs et formation à l'étayage dans le cadre d'une séquence de géométrie au CE2. École supérieure du professorat et de l'éducation, Académie de Nantes.

Baudoin, N. et Galand, B. (2018). Le climat scolaire influence-t-il le bienêtre des élèves ?. Dans N. Rousseau et N. Espinosa (dir.), Le bienêtre à l'école : enjeux et stratégies gagnantes (p. 15-30). Presses de l'Université du Québec.

Beckers, J. (2007). Compétences et identité professionnelles: l'enseignement et autres métiers de l'interaction humaine. De Boeck Supérieur. https://doi.org/10.3917/dbu.becke.2007.01

Beillerot, J. (1998). Formes et formations du rapport au savoir. L'Harmattan.

Bissonnette, D. (2020). L'impact des changements de pratiques pédagogiques chez les enseignants sur le développement des stratégies de lecture des élèves [mémoire de maitrise inédit, Université de l'Alberta].

Boutonnet, V. (2018). Usages et fonctions du matériel didactique: pratiques déclarées d'enseignants et de futurs enseignants d'histoire 


\section{$\&$}

\section{REVUE HYBRIDE DE L'ÉDUCATION}

au secondaire. Formation et profession, 26(2), 3-17. http://dx.doi.org/10.18162/fp.2018.462

Bozkurt, A. et Sharma, RC (2020). Enseignement à distance d'urgence en période de crise mondiale due à la pandémie de CoronaVirus. Asian Journal of Distance Education, 15(1), i-vi.

Bronfenbrenner, U. (1986). Dix années de recherche sur l'écologie du développement humain. Dans M. Crahay et $\mathrm{D}$. Lafontaine (dir.), L'art et la science de l'enseignement (p. 283-301). Éditions Labor.

Bucheton, D. (2009). L'agir enseignant : des gestes professionnels ajustés. Octares.

Bucheton, D. et Soulé, Y. (2009). Les gestes professionnels et le jeu des postures de l'enseignant dans la classe: un multi-agenda de préoccupations enchâssées. Éducation et Didactique, 3(3), 29-48. https://doi.org/10.4000/educationdidactique.543

Calkins, L. (2020). Teaching Writing. Heinemann.

Cinéus, J.-W. (2020). L'impact de la relation enseignant-élèves sur la réussite scolaire des élèves du secondaire en milieu socioéconomique faible au Québec: une recension critique des écrits [thèse de doctorat, Université de Sherbrooke]. Savoirs UdeS. https://savoirs.usherbrooke.ca/handle/11143/17661?show=full

Clark, R. Andrews, J. (2009). Peer mentoring in higher education: A literature review. Aston Centre for Learning Innovation and Professional Practice.

Dagenais-Desmarais, V. (2010). Du bien-être psychologique au travail : Fondements théoriques, conceptualisation et instrumentation du construit [thèse de doctorat, Université de Montréal]. Papyrus. https://papyrus.bib.umontreal.ca/xmlui/bitstream/handle/1866/3916/D agenais-

$\underline{\text { Desmarais Veronique } 2010 \text { these.pdf? sequence }=4 \& \text { is Allowed }=y}$

Florey, S. et Cordonier, N. (2017). Pour une place raisonnée de l'émotion dans la compréhension/interprétation et la réception des œuvres multimodales. Revue de recherches en littératie médiatique multimodale, 6. https://doi.org/10.7202/1043749ar

Gaudreau, N., Fortier, M.- P., Bergeron, G., Bonvin, P. et Vienneau, R. (2016). Gestion de classe et inclusion scolaire : pratiques exemplaires pour favoriser la réussite de tous. Dans L. Prud'homme, H. Duchesne et $P$. Bonvin (dir.), L'inclusion scolaire ses fondements, ses acteurs et ses pratiques (p. 139-152). De Boeck Supérieur. 


\section{REVUE HYBRIDE DE L'ÉDUCATION}

Gauthier, B. (2016). Recherche sociale : de la problématique à la collecte des données. Presses de l'Université du Québec.

Gouvernement de l'île-du-Prince-Édouard. (2006). La poésie, du jeu, des sons et des images. Ministère de l'Éducation et du Développement de la petite enfance. http://www.gov.pe.ca/photos/original/eecd poesieimag.pdf

Gouvernement du Québec (2020a). Organisation des activités scolaires 2020-2021

(COVID-19).

http://www.education.gouv.qc.ca/coronavirus/

Gouvernement du Québec (2020b). Référentiel des compétences professionnelles. Profession enseignante. Ministère de l'Éducation. https://cdn-contenu.quebec.ca/cdncontenu/adm/min/education/publications-adm/devenirenseignant/referentiel competences professionnelles profession e nseignante.pdf?1606848024

Goyette, N., Martineau, S., Gagnon, B. et Bazinet, J. (2020). Les effets d'une approche pédagogique préconisant la psychopédagogie du bienêtre sur la réussite éducative d'élèves du primaire. Revue hybride de l'éducation, 4(4), 1-23. https://doi.org/10.1522/rhe.v4i4

Hattie, J. (2017). L'apprentissage visible pour les enseignants : connaître son impact pour maximiser le rendement des élèves. Presses de l'Université du Québec.

Hodges, C., Moore, S., Lockee, B., Trust, T. et Bond, A. (2020). La différence entre l'enseignement à distance d'urgence et l'apprentissage en ligne. Revue Educause, 27. https://medicine.hofstra.edu/pdf/faculty/facdev/facdev-article.pdf

Lambert-Samson, V. (2016). L'anxiété des élèves au primaire : une analyse des connaissances théoriques et pratiques des enseignants [mémoire de maitrise, Université Laval]. Corpus. https://corpus.ulaval.ca/ispui/bitstream/20.500.11794/27048/1/32758. $\underline{\text { pdf }}$

Laurendeau, P. (2020, 13 mai). 5 éléments à considérer avant de se lancer dans l'enseignement à distance. École branchée. https://ecolebranchee.com/5-elements-avant-de-se-lancerenseignement-a-distance/

Lépine, M. (2017). L'enseignement de la lecture/appréciation des œuvres littéraires à l'école primaire : enquête sur les pratiques déclarées et les conceptions d'enseignants québécois [thèse de doctorat, Université 


\section{REVUE HYBRIDE DE L'ÉDUCATION}

de Montréal].

https://papyrus.bib.umontreal.ca/xmlui/handle/1866/20052

Papyrus.

Maubant, P. (2007). L'analyse des pratiques enseignantes : les ambiguïtés d'un bel objet de recherche. Formation et profession, 13(2), 17-21

Ministère de l'Éducation, du Loisir et du Sport. (2011). Le programme international de recherche en lecture scolaire (PIRLS) 2011 : résultats obtenus par les élèves québécois de 10 ans. Gouvernement du Québec. $\quad$ http://www.education.gouv.qc.ca/references/txsolrtyperecherchepublicationtx-solrpublicationnouveaute/resultatsde-la-recherche/detail/article/programme-international-de-rechercheen-lecture-scolaire-pirls-resultats-obtenus-par-leseleves/?a=a\&cHash=efbc2164bcd1e1ec467bdca3fd2d80a9

Morlaix, S. (2015). Les compétences sociales: quels apports dans la compréhension des différences de réussite à l'école primaire?. Hal Archives Découvertes. https://halshs.archives-ouvertes.fr/halshs$\underline{01111163}$

Paillé, P. et Mucchielli, A. (2016). L'analyse qualitative en sciences humaines et sociales ( $4^{\mathrm{e}}$ éd.). Armand Colin.

Papazian-Zohrabian, G. et Mamprin, C. (2020). L'école en temps de pandémie. Favoriser le bien-être des élèves et des enseignants. Université de Montréal. https://fse.umontreal.ca/fileadmin/fse/documents/pdf/publications/Gui de pandemie Final 16.09.2020.pdf

Paratte, L. (2006). La collaboration école-famille : motifs et pratiques des enseignants du préscolaire et du primaire dans la promotion de la participation des parents en classe [mémoire de maitrise, Université du Québec à Rimouski]. Semaphore. http://semaphore.uqar.ca/id/eprint/154/1/Lucie Paratte mars2006.pd $\underline{f}$

Potvin, M. C., Dion, E., Brodeur, M. et Borri-Anadon, C. (2012). Utiliser le tutorat par les pairs pour favoriser l'apprentissage de la lecture en milieu défavorisé. Une pré-expérimentation avec examen des caractéristiques des non-répondants. Revue canadienne de l'éducation, 35(2), 285-302.

Rasinski, T. V. et Padak, N. (2004). Effective reading strategies: Teaching children who find reading difficult ( $3^{\mathrm{e}}$ éd.). Pearson Merrill Prentice Hall 


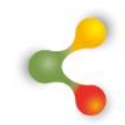

\section{REVUE HYBRIDE DE L'ÉDUCATION}

Reuter, Y. (2019). Didactiques, disciplines et interdisciplinarité. Dans F. Darbellay, M. Louviot et Z. Moody (dir.), L'interdisciplinarité à l'école. Succès, résistance, diversité (p. 133-150). Presses universitaires suisses.

Rezrazi, E. M. (2020). Peacekeeping missions in the time of coronavirus: New configurations and new challenges. Policy Center for the new South. https://covidaba.com/wpcontent/uploads/2020/06/Peacekeeping-Missions-in-the-Time-ofCoronavirus-New-Configurations-and-New-Challenges.pdf

Ribau, C., Lasry, J., Bouchard, L., Moutel, G., Hervé, C. et Marc-Vergnes, J. (2005). La phénoménologie: une approche scientifique des expériences vécues. Recherche en soins infirmiers, 81(2), 21 27. https://doi.org/10.3917/rsi.081.0021

Royer, C., Baribeau, C. et Duchesne, A. (2009). Les entretiens individuels dans la recherche en sciences sociales au Québec : où en sommesnous? Un panorama des usages. Recherches qualitatives, 7, 64-79.

Schwartz, S. (2016). Guide pour un enseignement durable au primaire (traduit par Y. Nadon). D'eux.

Simonin, L. (2019). La manipulation en tant que stratégie pédagogique : l'impact d'un élevage de phasme dans l'apprentissage des sciences [mémoire de maitrise, Université de Franche-Comte]. HAL. https://haluniv-fcomte.archives-ouvertes.fr/hal-02385056

Talbot, J. et Lessard, L. (2020). Revue de presse sur la santé mentale et la COVID-19: groupes ciblés et facteurs d'influence. Quintessence, 11(S5).

Thouin, M. (2014). Réaliser une recherche en didactique. Éditions Multimondes.

Organisation des Nations unies pour l'éducation, la science et la culture (UNESCO). (2020). Éducation : de la fermeture des établissements scolaires à la reprise. https://fr.unesco.org/covid19/educationresponse

Villeneuve-Lapointe, M., Beaulieu, J. et Vincent, F. (2018). Quelles pratiques différenciées pour l'enseignement de l'orthographe lexicale? Une recension des écrits. Canadian Journal of Education/Revue canadienne de l'éducation, 41(2), 498-523.

Villiot-Leclercq, E. (2020). L'ingénierie pédagogique au temps de la Covid19. Distances et médiations des savoirs, 30, 1-11. https://doi.org/10.4000/dms.5203 


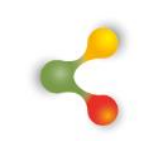

\section{REVUE HYBRIDE DE L'ÉDUCATION}

Vygotski, L. S. (1997). Pensée et langage (3éd.). La Dispute.

Wood, E. (2014). The play-pedagogy interface in contemporary debates. Dans L. Brooker, M. Blaise et S. Edwards (dir.), The SAGE handbook of play and learning in early childhood (p. 145-156). SAGE Publications. http://dx.doi.org/10.4135/9781473907850.n13 